\title{
ON THE LOCUS OF HODGE CLASSES
}

\author{
EDUARDO CATTANI, PIERRE DELIGNE, AND AROLDO KAPLAN
}

\section{INTRODUCTION}

Let $S$ be a complex algebraic variety and $\left\{X_{s}\right\}_{s \in S}$ a family of nonsingular projective varieties parametrized by $S$ : the $X_{s}$ are the fibers of $f: X \rightarrow S$, with $X$ projective and smooth over $S$. Fix $s \in S$, an integer $p$, and a class $h \in H^{2 p}\left(X_{s}, \mathbb{Z}\right)$ of Hodge type $(p, p)$. Let $U$ be an open, simply connected neighborhood of $s$. The $H^{2 p}\left(X_{t}, \mathbb{Z}\right), t \in S$, form a local system on $S$, necessarily trivial on $U$, so that for $t \in U$ they can all be identified with $H^{2 p}\left(X_{s}, \mathbb{Z}\right)$. The Hodge filtration $\mathscr{F}_{t}$ of $H^{2 p}\left(X_{t}, \mathbb{C}\right), t \in U$, can be viewed as a variable filtration on the fixed complex vector space $H^{2 p}\left(X_{s}, \mathbb{C}\right)$. It varies holomorphically with $t$. It follows that the locus $T \subset U$ where $h$ remains of type $(p, p)$, i.e., in $\mathscr{F}^{p}$, is a complex analytic subspace of $U$.

It follows from the (rational) Hodge conjecture that the germ of $T$ at $s$ is algebraic, meaning that its irreducible components are irreducible components of germs at $s$ of algebraic subvarieties of $S$. Sketch of proof: let $T^{0}$ be an irreducible component of $T$ containing $s$ and assume that for all $t$ in $T^{0}$ some nonzero multiple of $h$ is the class of an algebraic cycle in $X_{t}$. A Baire category argument shows that for suitable $M, N$, the set of $t \in T^{0}$ for which $h=\frac{1}{N}$ class of $\left(Z_{t}^{+}-Z_{t}^{-}\right)$, with $Z_{t}^{ \pm}$effective algebraic cycles of degree $\leq M$ on $X_{t}$, is dense in some nonempty open subset of $T^{0}$. One then uses that the Chow varieties of effective cycles of degree $\leq M$ on the $X_{t}$ form an algebraic variety over $S$, or simply that they form a limited family.

In Corollary 1.2 below, we prove unconditionally that the germ of $T$ at $s$, as above, is indeed algebraic. Our main result, Theorem 1.1, is slightly more precise and gives as corollary a positive answer to a question of A. Weil [7]: "... whether imposing a certain Hodge class upon a generic member of [such family] amounts to an algebraic condition upon the parameters."

The Hodge conjecture would. also imply that if $f: X \rightarrow S$ can be defined over an algebraically closed subfield of $\mathbb{C}$, then so can the germ of $T$ at $s$. About this, we are not able to say anything.

The proof will be in the setting of variations of Hodge structures, of which the local system of the $H^{2 p}\left(X_{t}, \mathbb{Z}\right)$ modulo torsion is an example. For the

Received by the editors February 8, 1994.

1991 Mathematics Subject Classification. Primary 14C25, 14C30, 14D07, 32G20, 32J25.

The first and third authors were partially supported by National Science Foundation Grant DMS-9107323. 
definition and basic properties of variations of Hodge structures on a complex variety $S$, we refer the reader to [2], [5], and [6].

Our notations are spelled out in 2.1. Note that in the Hodge decomposition, we do not assume $p, q \geq 0$. If $H$ is a Hodge structure of even weight $2 p$, shifting the Hodge filtration, i.e., replacing $H$ by the Tate twist $H(p)$, one obtains a Hodge structure of weight 0 . Classes of type $(p, p)$ become of type $(0,0)$. This allows us to restrict our attention to variations of weight 0 and classes of type $(0,0)$. For simplicity, we will assume the parameter space $S$ to be nonsingular.

Let $S$ be a nonsingular complex algebraic variety and $\mathscr{V}$ a variation of Hodge structures of weight 0 on $S$ with polarization form $Q$. If $\mathscr{C}$ is the Weil operator: multiplication by $i^{p-q}$ on $\mathscr{V}^{p, q}$, the hermitian form $h(u, v)=$ $Q(\mathscr{C} u, \bar{v})$ is positive definite and makes the Hodge decomposition orthogonal. For $u$ a real element of type $(0,0), h(u, u)=Q(u, u)$. Fix an integer $K$ and let $S^{(K)}$ be the space of pairs $(s, u)$ with $s \in S, u \in \mathscr{V}_{s}$ integral of type $(0,0)$, and $Q(u, u) \leq K$. It projects to $S$ and arguments as above show that, -locally on $S, S^{(K)}$ is a finite disjoint sum of closed analytic subspaces. Our main result is:

Theorem 1.1. $S^{(K)}$ is an algebraic variety, finite over $S$.

Corollary 1.2. Fix $s \in S$ and $u \in \mathscr{V}_{s}$ integral of type $(0,0)$. The germ of analytic subvariety of $S$ where $u$ remains of type $(0,0)$ is algebraic.

Proof. The required algebraic subvarieties of $S$ are images in $S$ of irreducible components of $S^{(K)}$, for $K=Q(u, u)$.

Corollary 1.3. Let $u$ be a section of the local system $\mathscr{V}_{\mathbb{Z}}$ on a universal covering of $S$. The set of points in $S$ where some determination of $u$ is of type $(0,0)$ is an algebraic subvariety of $S$.

Proof. Such a set is a union of images of connected components of $S^{(K)}$, for $K=Q(u, u)$.

Corollary 1.4. Let $\mathscr{V}$ be a polarizable variation of Hodge structures on $S$, fix $s \in S$, and let $U_{\mathbb{Q}} \subset\left(\mathscr{V}_{s}\right)_{\mathbb{Q}}$ be a rational subspace. The locus where some flat translate of $U_{\mathbb{Q}}$ is a Hodge substructure is an algebraic subvariety of $S$.

Proof. Let $T$ be the locus in question, assumed nonempty, and suppose first that $U_{\mathbb{Q}}$ is of dimension one. Then $\mathscr{V}$ is of even weight $2 p$ so that, replacing it by $\mathscr{V}(p)$, we may and shall assume it to be of weight 0 . Let $e$ be a generator of $U_{\mathbb{Q}} \cap \mathscr{V}_{\mathbb{Z}}$. Then $U_{\mathbb{Q}}$ is a Hodge substructure if and only if $e$ is of type $(0,0)$, and one applies 1.3.

Consider now the general case: $U_{\mathbb{Q}}$ of dimension $n$. A Hodge structure on a rational vector space $H_{\mathbb{Q}}$ gives rise to an action of the real algebraic group $\mathbb{C}^{*}$ on $H_{\mathbb{R}}=H_{\mathbb{Q}} \otimes \mathbb{R}$, with $z \in \mathbb{C}^{*}$ acting as multiplication by $z^{-p} \bar{z}^{-q}$ on $H^{p, q}$. A subspace $U_{\mathbb{Q}} \subset H_{\mathbb{Q}}$ is a sub-Hodge structure -i.e., $U_{\mathbb{C}}=U_{\mathbb{Q}} \otimes \mathbb{C}$ is the sum of its intersections with the $H^{p, q}$ - if and only if $U_{\mathbb{R}}$ is stable under $\mathbb{C}^{*}$. This amounts to $\bigwedge^{n} U_{\mathbb{R}} \subset \Lambda^{n} H_{\mathbb{R}}$ being stable under $\mathbb{C}^{*}$, i.e., to $\Lambda^{n} U_{\mathbb{Q}}$ being 
a Hodge substructure of $\wedge^{n} H_{\mathbb{Q}}$, and reduces us to the one-dimensional case, proving 1.4.

Let $D^{*}=D-\{0\}$ be the open punctured disk. Theorem 1.1 will be derived from the following local result.

Theorem 1.5. Let $\mathscr{V}$ be a polarized variation of Hodge structures of weight 0 on $S=D^{* r} \times D^{m}$, with unipotent monodromy. Define $S^{(K)}$ as in 1.1. Then, there is a neighborhood $U$ of 0 in $D^{r+m}$ such that, above $U, S^{(K)}$ is a finite disjoint sum of traces on $S \cap U$ of closed analytic subspaces of $U$.

Proof of $1.5 \Rightarrow 1.1$. To prove 1.1 one is free to replace $S$ of 1.1 by a finite étale covering $S^{\prime} \rightarrow S$. We may and shall assume that the monodromy mod $k$ of $\mathscr{V}$ is trivial, for some $k \geq 3$.

Let $\bar{S}$ be a smooth compactification of $S$, with $\bar{S}-S$ a divisor with normal crossings. The assumption on the monodromy ensures that the local monodromy of $\mathscr{V}$ at infinity is unipotent: in a neighborhood of any point in $\bar{S}-S$, one is in the situation considered in 1.5. One concludes that $S^{(K)}$ can be extended to a space $\bar{S}^{(K)}$ over $\bar{S}$, which locally over $\bar{S}$ is a finite disjoint sum of closed analytic subspaces. By GAGA, $\bar{S}^{(K)}$, finite over $\bar{S}$, is algebraic, and 1.1 follows.

The rest of the paper is devoted to proving 1.5. To simplify notations, we will assume $m=0$. The general case is recovered by considering the partition of $S$, according to which of $z_{r+1}, \ldots, z_{r+m}$ vanish.

We thank J. Carlson, H. Clemens, R. Donagi, V. Navarro Aznar, and J. Steenbrink for many useful conversations.

\section{Notations. SChEMA OF PROOF}

2.1. Let $\mathscr{V}$ be a polarized variation of Hodge structures of weight $w$ on a complex manifold $S$, with polarization form $Q$. We write $\mathscr{V}_{\mathbb{Z}}$ for the underlying local system of free $\mathbb{Z}$-modules, $\mathscr{V}_{\mathscr{\theta}}$ for the corresponding holomorphic vector bundle, identified with its sheaf of sections $\mathscr{O}_{S} \otimes \mathscr{V}_{\mathbb{Z}}$, and $\mathscr{V}$ for the underlying complex vector bundle, identified with its sheaf of $C^{\infty}$ sections. The Hodge decomposition is a decomposition of the complex vector bundle

$$
\mathscr{V}=\bigoplus_{p+q=w} \mathscr{V}^{p, q},
$$

and the corresponding Hodge filtration

$$
\mathscr{F}^{p}=\bigoplus_{a \geq p} \mathscr{V}^{a, b}
$$

is holomorphic. We still write $\mathscr{F}^{p}$ for the corresponding filtration of $\mathscr{V}_{\mathscr{O}}$. The Griffiths transversality axiom then reads

$$
\nabla \mathscr{F}^{p} \subset \Omega^{1} \otimes \mathscr{F}^{p-1} .
$$

The Weil operator $\mathscr{C}$ is the endomorphism of $\mathscr{V}$ acting on $\mathscr{V}^{p, q}$ as multiplication by $i^{p-q}$. The polarization form $Q$ is best viewed as a morphism 
of Hodge structure $\mathscr{V} \otimes \mathscr{V} \rightarrow \mathbb{Z}(-w)$. It is an integral bilinear form on $\mathscr{V}_{\mathbb{Z}}$, $(-1)^{w}$-symmetric, and the form on $\mathscr{V}$

$$
h(u, v)=Q(\mathscr{C} u, \bar{v})
$$

is hermitian symmetric, positive definite, and makes the Hodge decomposition orthogonal. This Hodge metric is, generally, not flat.

2.2. We will need Schmid's theory of nilpotent orbits giving the asymptotic behavior of variations of Hodge structures [6]. We begin with a coordinatefree description. The coordinate-bound translation given in 2.3 suffices for our needs.

Let $S$ be the complement in a smooth variety $\bar{S}$ of smooth divisors $E_{i}$ meeting transversally. Assume that the monodromy of $\mathscr{V}$ around the $E_{i}$ is unipotent. Let $\mathscr{V}_{\mathscr{O}}^{-}$be the canonical extension [4] of the holomorphic vector bundle $\mathscr{V}_{\mathscr{O}}$ to $\bar{S}$. It is characterized by the property that, in any local basis of $\mathscr{V}_{\mathscr{O}}^{-}$, the connection matrix (an endomorphism-valued 1-form) has logarithmic poles with nilpotent residues along the $E_{i}$. The first result is that

(2.2.1) The Hodge filtration $\mathscr{F}$ of $\mathscr{V}_{\mathscr{O}}$ extends to a filtration of $\mathscr{V}_{\mathscr{O}}^{-}$by locally direct factors.

Let $E$ be the intersection of the $E_{i}, L_{i}$ the restriction to $E$ of the normal line bundle of $E_{i}$, and $L_{i}^{*}$ the complement in $L_{i}$ of the zero section. The product $L$ of the $L_{i}$ is the normal bundle of $E$, and the product $L^{*} \subset L$ of the $L_{i}^{*}$ is obtained by removing from the normal bundle $L$ the normal bundles of $E$ in the $E_{i}$.

The nilpotent orbit $\mathscr{V}_{\text {un }}$ (or: deformation to the normal cone) approximating $\mathscr{V}$ around $E$ lives on $L^{*}$. It is obtained as follows:

(a) As a filtered holomorphic vector bundle, it is the pull back of the restriction of $\left(\mathscr{V}_{\mathscr{O}}^{-}, \mathscr{F}\right)$ to $E$.

(b) The connection $\nabla_{\text {un }}$ on $\mathscr{V}_{\text {un }}$ is described as follows. Locally, let $z_{i}=0$ be an equation for $E_{i}$. It defines a trivialization of $L_{i}$ and we write again $z_{i}$ for the corresponding function on $L$. In a local basis of $\mathscr{V}_{\mathscr{O}}^{-}$, the connection $\nabla$ is $d+\Gamma$, with $\Gamma=\Gamma_{0}+\sum \nu_{i} \frac{d z_{i}}{z_{i}}, \Gamma_{0}$ and $\nu_{i}$ holomorphic. The local basis of $\mathscr{V}_{\mathscr{O}}^{-}$gives one on $E$, which pulls back to $\mathscr{V}_{\text {un }}$; in this basis,

$$
\nabla_{\mathrm{un}}=d+\left(\Gamma_{0} \mid E\right)+\sum\left(\nu_{i} \mid E\right) \frac{d z_{i}}{z_{i}} .
$$

(c) It remains to define the integral lattice. Locally, let $\phi$ be an isomorphism from a neighborhood of the zero section of $L$ (identified with $E$ ) to a neighborhood of $E$ in $\bar{S}$. Assume it is the identity on $E$ and that, on $E, d \phi$ induces the identity on the normal bundle of $E$. Assume further that $\phi$ maps $L^{*}$ into $S$. Then, the pull back by $\phi$ of the local system $\mathscr{V}^{\nabla}$ of horizontal sections of $\mathscr{V}$ is canonically isomorphic to $\mathscr{V}_{\text {un }}^{\nabla}$. One defines $\left(\mathscr{V}_{\text {un }}\right)_{\mathbb{Z}}$ to be the pull back of $\mathscr{V}_{\mathbb{Z}}$.

The results are that 
(2.2.2) On the trace on $L^{*}$ of a neighborhood $U$ of $E$, with $U$ depending only on the rank of $\mathscr{V}, \mathscr{V}_{\text {un }}$ is a variation of Hodge structures, polarized by a polarization form of $\mathscr{V}$.

(2.2.3) For $\phi$ as in (c), defined on $U, \mathscr{V}_{\text {un }}$ and $\phi^{*} \mathscr{V}$ are close: on any compact $K \subset U$, the distance at $z \in K \cap L^{*}$ between the Hodge filtrations, measured with an invariant metric on the relevant period mapping domain, i.e., using the Hodge metrics, is

$$
\leq C_{1} d(z, E)|\log d(z, E)|^{C_{0}}
$$

with $C_{0}$ depending only on the rank, and $C_{1}$ only on the rank, on $K$, and on the chosen distance $d$.

2.3. We now translate in coordinates. Let $D \subset \mathbb{C}$ be the open unit disk and $D^{*}=D-\{0\}$. Take $S=D^{* r} \subset D^{r}$. Assume that the monodromy of $\mathscr{V}$ is unipotent. Let $M_{i}$ be the monodromy around $z_{i}=0$ and

$$
N_{i}=-\log M_{i} \text {. }
$$

Using the coordinate $z_{i}$, one can view $\mathscr{V}_{\text {un }}$ as living on $\mathbb{C}^{* r} \supset S$. The underlying local system is the unique local system extending $\mathscr{V}_{\mathbb{Z}}$ on $S$. We continue to denote it by $\mathscr{V}_{\mathbb{Z}}$. Let $V_{\mathbb{Z}}$ be its fiber at 1 .

The Poincare upper half-plane $\mathscr{H}$ is the universal covering of $D^{*}$, with covering map $z \mapsto s=e^{2 \pi i z}$. Similarly, $\mathscr{H}^{r}$ is the universal covering of $S=D^{* r}$, and $\left(\mathbb{C}^{r}, 0\right)$ that of $\left(\mathbb{C}^{* r}, 1\right)$. For $z \in \mathbb{C}^{r}$, we write $s$ for the corresponding point in $\mathbb{C}^{* r}$.

When pulled back to $\mathscr{H}^{r}$, the variation can be described as a variable Hodge filtration $\Phi(z)$ on the fixed vector space $V=V_{\mathbb{Z}} \otimes \mathbb{C}$, with

$$
\Phi\left(z+e_{j}\right)=\exp \left(N_{j}\right) \Phi(z)
$$

for $e_{j}$ the $j^{\text {th }}$ coordinate vector in $\mathbb{C}^{r}$. We also view $\Phi$ as a holomorphic map $\Phi: \mathscr{H}^{r} \rightarrow \mathscr{D}\left(V_{\mathbb{Z}}, Q\right)$ with values in the appropriate period mapping domain.

Statement (2.2.1) translates as

$$
\Phi(z)=\exp \left(\sum z_{j} N_{j}\right) \Psi(s)
$$

$\left(s=e^{2 \pi i z}\right)$ for some holomorphic map $\Psi$ from $D^{r}$ to the flag manifold of $V$. The nilpotent orbit $\mathscr{V}_{\text {un }}$ is given by

$$
\Phi_{\mathrm{un}}(z)=\exp \left(\sum z_{j} N_{j}\right) \Psi(0) .
$$

Statements (2.2.2) and (2.2.3) translate as the existence of constants $C_{0}, C_{2}$, $C_{3}$, depending only on the rank of $\mathscr{V}$, with the following properties. Let $x_{i}$ and $y_{i}$ be the real and imaginary parts of $z_{i}$. Then, as soon $\operatorname{as} \inf \left(y_{i}\right) \geq C_{2}$,

(2.3.2) $\Phi_{\mathrm{un}}(z)$ defines a Hodge structure on $V$.

(2.3.3) The distance between $\Phi(z)$ and $\Phi_{\mathrm{un}}(z)$, measured as in (2.2.3), is

$$
\leq C_{3} e^{-2 \pi \inf \left(y_{j}\right)} \inf \left(y_{j}\right)^{C_{0}} .
$$


Fix $I \subset\{1, \ldots, r\}$, with complement $J$. Let $E$ be the intersection in $D^{r}$ of the divisors $z_{i}=0$, for $i \in I$. It is identified with $D^{J}$. Let $q_{I}$ be the projection $D^{* r} \rightarrow D^{* J}$. The nilpotent orbit $\mathscr{V}_{\text {un }, I}$ along $E$ is then given by

$$
\Phi_{\text {un }, I}(z)=\exp \left(\sum z_{j} N_{j}\right) \Psi\left(q_{I}(s)\right) .
$$

Freezing the $z_{j}(j \in J)$ and applying (2.3.2), (2.3.3), one sees that $\Phi_{\mathrm{un}, I}(z)$ gives a Hodge structure on $V$ as soon as $\inf _{i \in I}\left(y_{i}\right) \geq C_{2}$, and that the proximity of $\Phi_{\mathrm{un}, I}(z)$ to $\Phi(z)$ is controlled by $\inf _{i \in I}\left(y_{i}\right)$ as in (2.3.3).

2.4. Any nilpotent endomorphism $N$ of $V, N^{k+1}=0$, has an associated filtration $W(N)$. This is an increasing filtration

$$
\{0\}=W_{-k-1}(N) \subset \cdots \subset W_{k}(N)=V
$$

characterized by the properties that

$$
N W_{\ell} \subset W_{\ell-2}
$$

and that $N^{\ell}$ induces an isomorphism

$$
N^{\ell}: G r_{\ell}^{W} \stackrel{\sim}{\rightarrow} G r_{-\ell}^{W} \text {. }
$$

With the notations of 2.3 , it is shown in [1] that all elements $N$ in the cone

$$
C=\left\{\sum \lambda_{j} N_{j}, \lambda_{j}>0\right\}
$$

define the same filtration $W(C)$. As $C$ contains endomorphisms defined over $\mathbb{Q}$, the filtration $W:=W(C)$ is defined over $\mathbb{Q}$.

Let us call the "limiting Hodge filtration" any filtration of the form $\Phi_{\mathrm{un}}(z)$ $\left(z \in \mathbb{C}^{r}\right)$. It is a consequence of the $S L(2)$-orbit theorem [6] that, for any limiting Hodge filtration $F, F$ and $W[-w]$ define a mixed Hodge structure. Here $w$ is the weight of $\mathscr{V}$ and $W[-w]_{\ell}=W_{\ell-w}$. The mixed Hodge structure $(W[-w], F)$ is polarized, in the sense of [1], by the polarization form of $\mathscr{V}$ and any $N$ in $C$.

Theorem 1.5 will be deduced from the following result, where we use the notations of 2.3, 2.4.

Theorem 2.5. Assume $\mathscr{V}$ of weight 0 . Given $K$, there is a constant $A_{1}$ (depending on $K$ and $\mathscr{V}$ ) such that

(i) There are only finitely many $v \in V_{\mathbb{Z}}$ such that: $Q(v, v) \leq K$ and $v \in \Phi^{0}(z)$ at some point $z$ with $0 \leq x_{i} \leq 1$ and $\inf \left(y_{i}\right) \geq A_{1}$.

(ii) Any such $v$ is in $W_{0}$, as well as in $F_{v}^{0}$ for some limiting Hodge filtration $F_{v}$.

Remark 2.6. If $v$ is, at $z$, in $\Phi^{0}$, it defines a morphism of Hodge structures from the unit Hodge structure $\mathbb{Z}(0)$ to $\mathscr{V}$ at $z$. By (ii), if $Q(v, v) \leq K$ and $\inf \left(y_{i}\right) \geq A_{1}$, it will also define a morphism of mixed Hodge structures from $\mathbb{Z}(0)$ to $\mathscr{V}_{\text {un }}$ at some point $z^{\prime}$. In 2.13 , we will deduce from 2.5 that $z^{\prime}$ can be chosen such that

with $A_{2}$ depending only on $\mathscr{V}$.

$$
\left|z^{\prime}-z\right| \leq A_{2} e^{-2 \pi \inf \left(y_{i}\right)}
$$


2.7. As a preparation to deducing 1.5 from 2.5 , we now fix $v \in V_{\mathbb{Z}}$ which, for some $z_{o}$, is in $W_{0} \cap \Phi_{\text {un }}^{0}\left(z_{o}\right)$, and investigate the locus where $v \in \Phi^{0}(z)$. We make the change of variables $z \mapsto z-z_{o}, s \mapsto s / s_{o}$, to simplify notations by assuming $z_{o}=0$. This is at the cost of having $\Phi(z)$ defined, in the new coordinates, only for $y_{i} \geq-\Im\left(z_{o i}\right)$.

Let $F$ be the filtration $\Phi_{\mathrm{un}}(0)$. Let $V^{p, q}$ be the bigrading of $V$ associated to the mixed Hodge structure $(W, F)$ (cf. $[3,2.13])$. It splits the filtrations $W$ and $F: W_{\ell}\left(\right.$ resp. $\left.F^{p}\right)$ is the sum of the $V^{a, b}$ for $a+b \leq \ell$ (resp. $a \geq p$ ). The assumptions on $v$ imply that $v \in V^{0,0}$.

The Lie algebra $\mathfrak{g}=\mathfrak{g l}(V)$ of $G L(V)$ inherits from $V$ a mixed Hodge structure whose associated bigrading is

$$
\mathfrak{g}^{p, q}=\left\{X \in \mathfrak{g} \mid X V^{r, s} \subset V^{p+r, q+s}\right\} .
$$

We will mainly use the corresponding $p$-grading, for which $V^{p}$ (resp. $\mathfrak{g}^{p}$ ) is the sum of the $V^{p, b}$ (resp. $\mathfrak{g}^{p, b}$ ).

The isotropy subalgebra of $\mathfrak{g}$ at $F$ is $F^{0}(\mathfrak{g})=\bigoplus_{p \geq 0} \mathfrak{g}^{p}$. It admits as supplement the nilpotent subalgebra $\mathfrak{b}=\bigoplus_{p<0} \mathfrak{g}^{p}$. The map $X \mapsto \exp (X) F$ identifies a neighborhood of 0 in $\mathfrak{b}$ with a neighborhood of $F$ in the flag space. As $\Psi(0)=F$, we can, for $y_{i}$ large enough, rewrite (2.3.1) as

$$
\Phi(z)=\exp \left(\sum z_{j} N_{j}\right) \exp (\Gamma(s)) F
$$

$\left(s=e^{2 \pi i z}\right.$ ) with $\Gamma$ holomorphic at $s=0, \mathfrak{b}$-valued, and such that $\Gamma(0)=0$.

The $N_{j}$ are morphisms of mixed Hodge structures $V \rightarrow V(1)$, hence they lie in $\mathfrak{g}^{-1,-1} \subset \mathfrak{g}^{-1} \subset \mathfrak{b}$. As $v$ is in $V^{0}$, the equation

$$
v \in \Phi^{0}(z)=\exp \left(\sum z_{j} N_{j}\right) \exp (\Gamma(s)) F^{0}
$$

or, equivalently,

$$
\left(\exp \left(\sum z_{j} N_{j}\right) \exp (\Gamma(s))\right)^{-1}(v) \in F^{0}=\bigoplus_{p \geq 0} V^{p}
$$

holds if and only if $v$ is fixed by $\exp \left(\sum z_{j} N_{j}\right) \exp (\Gamma(s))$. Let $\Gamma_{p}(s)$ be the component of $\Gamma(s)$ in $\mathfrak{g}^{p}$. Taking the component in $V^{-1}$ of the equation $\exp \left(\sum z_{j} N_{j}\right) \exp (\Gamma(s))(v)=v$, we obtain

$$
\left(\sum z_{j} N_{j}+\Gamma_{-1}(s)\right)(v)=0 .
$$

We now use the transversality axiom to prove:

Lemma 2.8. If at one point of the analytic space $\Sigma$ where (2.7.3) holds one has $v \in \Phi^{0}(z)$, the same is true on the whole connected component of $\Sigma$ containing that point.

Proof. Equation (2.7.3) is equivalent to

$$
\left(\exp \left(\sum z_{j} N_{j}\right) \exp (\Gamma(s))\right)^{-1}(v) \in F^{0} \oplus \bigoplus_{p \leq-2} V^{p},
$$


i.e., to

$$
v \in \exp \left(\sum z_{j} N_{j}\right) \exp (\Gamma(s))\left(F^{0} \oplus \bigoplus_{p \leq-2} V^{p}\right),
$$

so that 2.8 is a particular case of

Lemma 2.9. Let $\mathscr{X}$ be a variation of Hodge structures on a complex manifold $M$. Let $\mathscr{U}$ be a supplement to $\mathscr{F}^{p-1}$. For $v$ a horizontal section of $\mathscr{X}$, let $\Sigma$ be the locus where $v \in \mathscr{F}^{p}+\mathscr{U}$. Then, if at one point of $\Sigma$ one has $v \in \mathscr{F}^{p}$, then the same holds in the whole connected component of $\Sigma$ containing that point.

Proof. By assumption, $\mathscr{X}=\mathscr{F}^{p-1} \oplus \mathscr{U}$. The connection $\nabla$ of $\mathscr{X}$ induces a connection $\nabla_{\mathscr{U}}$ on $\mathscr{U}$, not necessarily integrable:

$$
\nabla_{\mathscr{U}}(\xi)=\text { projection of } \nabla(\xi) \text {. }
$$

Let $\hat{v}$ be the projection of $v$ to $\mathscr{U}$. Because $\nabla \mathscr{F}^{p} \subset \Omega^{1} \otimes \mathscr{F}^{p-1}$ on $\Sigma, \hat{v}$ is a horizontal section of $\mathscr{U}$. The lemma follows.

2.10. By 2.8, the locus where $v \in \Phi^{0}(z)$ is a union of connected components of the locus $\Sigma$ where (2.7.3) holds. Each $N_{j} v$ is in $V_{\mathbb{Q}}$. Writing (2.7.3) in a basis of $V_{\mathbb{Q}}$, we obtain a system of equations

$$
\sum \nu_{j}^{(\alpha)} z_{j}+\gamma^{(\alpha)}(s)=0 \quad\left(\alpha=1, \ldots, \operatorname{dim} V_{\mathbb{Q}}\right)
$$

with $\nu_{j}^{\alpha}$ rational, $\gamma^{(\alpha)}(s), s=e^{2 \pi i z}$, holomorphic at 0 , and $\gamma^{(\alpha)}(0)=0$.

Lemma 2.11. Near 0 in $D^{r}$ there is a closed analytic subspace $\Delta$ such that, if $\Sigma^{o}$ is a connected component of $\Sigma$ (equation (2.7.3)), its image in $D^{* r}$ is a connected component $\Delta^{o}$ of $\Delta \cap D^{* r}$. Further, the image of $\pi_{1}\left(\Delta^{o}\right)$ in $\pi_{1}\left(D^{* r}\right)$ fixes $v$.

Proof. If we clear denominators and exponentiate, equations (2.10.1) give

$$
a^{\alpha}(s) \prod s_{i}^{n(\alpha, i)}=1
$$

with $a^{\alpha}(s)$ holomorphic at $0, a^{\alpha}(0)=1$, and $n(\alpha, i) \in \mathbb{Z}$. That $a^{\alpha}(0)=1$ springs from the normalization $z_{0}=0$. As equations for $\Delta$, we take

$$
a^{\alpha}(s) \prod_{n(\alpha, i) \geq 0} s_{i}^{n(\alpha, i)}-\prod_{n(\alpha, i)<0} s_{i}^{-n(\alpha, i)}=0 .
$$

An element $m$ of $\pi_{1}\left(D^{* r}\right)=\mathbb{Z}^{r}$ is in the image of $\pi_{1}\left(\Delta^{o}\right)$ if and only if $\Sigma^{o}$ contains, together with any point $z$, the point $z+m$. Subtracting the corresponding equations $(2.10 .1)$, we obtain

$$
\sum \nu_{j}^{\alpha} m_{j}=0
$$

i.e.,

$$
\sum m_{i} N_{i} v=0
$$

Since $m$ acts on $V$ as $\exp \left(\sum m_{i} N_{i}\right)$, the lemma follows. 
2.12. Proof of $2.5 \Rightarrow 1.5$. As explained after 1.5 , it suffices in 1.5 to consider the case $m=0: S=D^{* r}$. By 2.5, 2.8, 2.11, there are, in a neighborhood of $0 \in D^{r}$, finitely many closed analytic subspaces $Y$ such that all connected components of $S^{(K)}$ are obtained by taking a connected component of $Y \cap D^{* r}$ and a section of $V_{\mathbb{Z}}$ on it.

2.13. Proof of 2.6. Expressed in $D^{* r}, 2.6$ claims that, for $s$ close enough to 0 , if $v \in(\mathscr{V})_{s}$ is of type $(0,0)$ and satisfies $Q(v, v) \leq K$, then at a nearby point $s^{\prime}$,

$$
\left|\frac{s^{\prime}}{s}-1\right| \leq A_{3}|s|
$$

the same $v$ is of type $(0,0)$ for $\mathscr{V}_{\text {un }}$. "Same" means: horizontal translate by a path remaining in the neighborhood (2.13.1) of $s$.

As the strip $0 \leq x \leq 1$ covers $D^{*}$, working on $\mathscr{C}^{r}$ we may assume that $v$ is as in 2.5. By 2.5 (i), we may treat those $v$ one at a time. We make the same change of variable $z \mapsto z-z_{o}$ as in 2.7. The equation (2.7.3) gives, for a fixed norm on $V$,

This implies that

$$
\left|\sum z_{j} N_{j} v\right| \leq A_{4} e^{-2 \pi \inf \left(y_{j}\right)} .
$$

$$
\begin{gathered}
\sum z_{j}^{\prime} N_{j} v=0, \text { with } \\
\left|z_{j}^{\prime}-z_{j}\right| \leq A_{5} e^{-2 \pi \inf \left(y_{j}\right)}:
\end{gathered}
$$

take $z^{\prime}-z$ to be the image of $-\sum z_{j} N_{j} v$ by a fixed linear section of $\mathbb{C}^{r} \rightarrow V$; $z \mapsto \sum z_{j} N_{j} v$. The condition $\sum z_{j}^{\prime} N_{j} v=0$ is equivalent to $v \in \Phi_{\mathrm{un}}^{0}\left(z^{\prime}\right)$.

Remark 2.14. The following example shows that the constant $A_{1}$ in 2.5 does depend on $\mathscr{V}$. Consider the case of a variation $\mathscr{V}$ on $D^{*}$ that extends to a variation on $D$. The nilpotent orbit $\mathscr{V}_{\text {un }}$ is then the constant variation with value $\mathscr{V}_{0}$. Fix a variation $\mathscr{W}$ on a bigger disk and $v \in \mathscr{W}_{\mathbb{Z}}$ of type $(0,0)$ at 0 and nowhere else. Translating $\mathscr{W}$ by any small $\epsilon$, we obtain $\mathscr{V}$ on $D$ and $v \in \mathscr{V}_{\mathbb{Z}}$ of bounded norm and type $(0,0)$ at $\epsilon$, but not at 0 , and hence nowhere for $\mathscr{V}_{\text {un }}$.

Theorem 2.5 is really a statement about what happens when there is a sequence of points $s^{\alpha} \in D^{* r}$ tending to 0 in $D^{r}$ and of $v^{\alpha} \in \mathscr{V}_{s^{\alpha}}$ integral of type $(0,0)$ and of bounded norm.

2.15. Heuristics for 2.5. Our method of proof forces us to prove a result more general than 2.5, where the assumption $v \in \Phi^{0}(z)$ is replaced by the assumption that $v$ is close to $\Phi^{0}(z)$.

In any hermitian space, given $\alpha$, a quantity $Y$, a nonzero vector $v$, and a subspace $F$, we will write

$$
v \sim_{Y} F
$$

if the sine of the angle between $v$ and $F$ is bounded by $\exp (-\alpha Y)$, i.e., if $v+w \in F$ with

$$
|w| \leq \exp (-\alpha Y)|v| \text {. }
$$


For $z=\left(z_{1}, \ldots, z_{r}\right)$, with imaginary part $y$, we will write $\sim_{z}$ for $\sim_{\sup \left(y_{i}\right)}$. We will denote by $\|v\|_{\Phi(z)}$ (or, simply, by $\|v\|$ if no ambiguity is possible) the Hodge norm of $v \in V$ at the point $\Phi(z)$.

Theorem 2.16. Assume $\mathscr{V}$ of weight 0 . Given $K$ and $\alpha>0$, there is a constant $A_{1}$ (depending on $K, \alpha$, and $\mathscr{V}$ ) such that

(i) There are only finitely many elements $v \in V_{\mathrm{Z}}$ such that, at some point $z$ with $0 \leq x_{i} \leq 1$ and $\inf \left(y_{i}\right) \geq A_{1},\|v\|_{\Phi(z)}^{2} \leq K$ and, relative to the Hodge metric at $\Phi(z)$,

$$
v \sim_{z} \Phi^{0}(z)
$$

(ii) Any such $v$ is in $W_{0}$.

(iii) If a fixed $v$ satisfies (i) at a sequence of points $z$ with $0 \leq x_{i} \leq 1$ and $\inf \left(y_{i}\right) \rightarrow \infty$, then $v$ is in $F^{0}$ for some limiting Hodge filtration $F$.

2.17. Remarks. (i) The proof could be strengthened to show that if $\mathscr{V}$ depends continuously on a parameter $\tau$ varying in a compact space, the constant $A_{1}$ can be taken independent of $\tau$.

(ii) In (2.16.1), we use the Hodge metric at $\Phi(z)$. We could as well have used a fixed metric. Indeed, the ratio between a fixed metric and the Hodge metric is bounded by a power of $\sup \left(y_{i}\right)$ (see (3.8 (i))) so that for any $\alpha^{\prime}<\alpha$ and for $\inf \left(y_{i}\right)$ large enough, $v \sim_{z} \Phi^{0}(z)$ for $\alpha$ and one metric implies $v \sim_{z} \Phi^{0}(z)$ for $\alpha^{\prime}$ and the other metric.

(iii) The condition $v \sim_{z} \Phi^{0}(z)$ implies that the ratio $Q(v, v) /\|v\|_{\Phi(z)}^{2}$ is close to one. Instead of the condition $\|v\|_{\Phi(z)}^{2} \leq K$, we could as well have required $Q(v, v) \leq K$.

2.18. To prove 2.16, one would like to be able to replace $\Phi$ by $\Phi_{\text {un }}$. For $\inf \left(y_{i}\right)$ large, $\Phi(z)$ and $\Phi_{\mathrm{un}}(z)$ are close - roughly at a distance $\exp \left(-2 \pi \inf \left(y_{i}\right)\right)$. The case of a variation extending across $D^{r}$ shows that one cannot hope for anything better. On the other hand, $\exp \left(\sum z_{i} N_{i}\right)$ is of $\operatorname{size} \sup \left(y_{i}\right)^{k}, k$ bounded by the rank of $\mathscr{V}$. If the $y_{i}$ are of wildly different magnitudes, the product

$$
\exp \left(-2 \pi \inf \left(y_{i}\right)\right) \cdot \sup \left(y_{i}\right)^{k}
$$

need not be small. This leads to difficulties which may be circumvented as follows.

Fix $I \subset[1, r]$, with complement $J$. Assume that $z$ is such that the $y_{i}$ $(i \in I)$ are of comparable size, and much bigger than the $y_{j}(j \in J)$. Let $W^{1}$ be the filtration attached, as in (2.4), to the elements of the cone

$$
C^{1}=\left\{\sum_{i \in I} \lambda_{i} N_{i}, \lambda_{i}>0\right\} .
$$

If we freeze the variables $z_{j}(j \in J)$ and consider the asymptotic nilpotent orbit of the resulting variations on $D^{* I}$, we obtain $\Phi_{\mathrm{un}, I}(z)$ which is close to $\Phi(z)$, with a proximity controlled by $\inf _{i \in I}\left(y_{i}\right)$. These nilpotent orbits, for 
variable $z_{j}(j \in J)$, fit into a period map $\Phi_{\mathrm{un}, I}$. For $z$ as above, a small angle between $v$ and $\Phi^{0}(z)$, as in (2.16.1), implies a similarly small angle $v$ and $\Phi_{\mathrm{un}, I}^{0}(z)$, as $\inf _{i \in I}\left(y_{i}\right) \sim \sup _{i \in[1, r]}\left(y_{i}\right)$ by assumption.

The first step will be to show that $v$ is in $W_{0}^{1}$. This cannot be viewed as a consequence of the variant 2.17 (i) of 2.16 (ii) with parameters, for variations on $D^{* I}$, as the required set $D^{* J}$ of parameters is noncompact. We have to rely on the $S L(2)^{r}$-orbit theorem [3], which controls $\Phi(z)$ in the whole of regions of the form $y_{1} \geq a y_{2}, y_{2} \geq a y_{3}, \ldots, y_{n-1} \geq a y_{r}$. Next, one shows that $\left(\sum_{i \in I} y_{i} N_{i}\right)(v)$ is small, and this allows one to find $z^{*}$, with the same $y_{j}$ $(j \in J)$ and with $y_{i}^{*}$ comparable to $\sup _{j \in J}\left(y_{j}\right)$ such that $v$ is close to $\Phi_{\mathrm{un}, I}^{0}$ at $z^{*}$, with a proximity controlled by $\inf _{i \in I}\left(y_{i}\right) \sim \sup _{i \in[1, r]}\left(y_{i}\right)$. Iterating this process, one eventually finds $z^{* *}$, with all $y_{i}^{* *}$ comparable to inf $\left(y_{i}\right)$, such that $v$ is close to $\Phi_{\mathrm{un}}^{0}\left(z^{* *}\right)$ (in the sense of (2.16.1), possibly for a new $\alpha$ ). The next step gives $v \in W_{0}$ and $v$ close to $\Phi_{\text {un }}^{0}\left(z^{* * *}\right)$, with $z^{* * *}$ bounded and a proximity controlled by $\inf \left(y_{i}\right)$. From this 2.16 follows.

2.19. To ease the handling of quantifiers and estimates, but at the cost of effectivity, we will prove 2.16 by contradiction. If (i) fails, we can find sequences $u(n), z(n)$ with the $(u(n), z(n))$ as in $2.16(\mathrm{i})$, the $u(n)$ all distinct, and $\inf _{i}\left(y_{i}(n)\right) \rightarrow \infty$. If (ii) fails, we can find sequences as above with each $u(n)$ not in $W_{0}$. If (i) and (ii) hold but (iii) fails, we can find similar sequences with $u(n)$ constant and $u(n)$ not in $F^{0}$ for any limiting Hodge filtration $F$. In each case, a subsequence of the offending sequence is again offending. To prove 2.16 by contradiction, it hence suffices to show that given a sequence $(u(n), z(n))$ with $u(n) \in V_{\mathbb{Z}},\|u(n)\|_{\Phi(z(n))}^{2} \leq K$, and $u(n) \sim_{z} \Phi^{0}(z(n))$ in the Hodge norm at $z(n), 0 \leq x_{i}(n) \leq 1, \inf \left(y_{i}(n)\right) \rightarrow \infty$, it has a subsequence for which $u(n)$ is constant, in $W_{0}$, and in $F^{0}$ for some limiting Hodge filtration $F$.

\section{Preliminaries}

In 3.2, we comment on what it means and what it takes for a mixed Hodge structure to be close to another. We then recall results of the $S L(2)^{r}$-theory of [3] in a form suitable for our purposes.

3.1. Let $V$ be a complex vector space. Fix subspaces $A, B$ of $V$. If subspaces $A^{\prime}, B^{\prime}$ are close to $A, B$ in their respective grassmannians, then

$$
\operatorname{dim} A^{\prime} \cap B^{\prime} \leq \operatorname{dim} A \cap B
$$

(upper semicontinuity of the map $(A, B) \mapsto \operatorname{dim} A \cap B)$. Further, on the space of pairs of subspaces $A, B$ with $A \cap B$ of fixed dimension, the map $(A, B) \mapsto$ $A \cap B$ is continuous.

Indeed, let $A^{\prime}, B^{\prime}$ be close to $A, B$. Some $g \in G L(V)$, close to the identity, maps $A^{\prime}$ to $A$ : we may and shall assume that $A^{\prime}=A$. Fix a supplement $C_{1}$ of $A \cap B$ in $A$ and a supplement $C_{2}$ of $A+B$ in $V:$ for $C=C_{1} \oplus C_{2}$, we have $V=B \oplus C$ and $A=(A \cap B) \oplus(A \cap C)$. Being close to $B, B^{\prime}$ is the graph of a map $b^{\prime}: B \rightarrow C$ with $B^{\prime} \mapsto b^{\prime}$ continuous. An element $u+b^{\prime}(u)$ of $B^{\prime}$ is 
in $A$ if and only if both $u \in B$ and $b^{\prime}(u) \in C$ are. This requires $u \in A \cap B$ and the assertion follows. If $\operatorname{dim} A \cap B^{\prime}=\operatorname{dim} A \cap B$, then $A \cap B^{\prime}$ is the graph of the restriction of $b^{\prime}$ to $A \cap B$, continuous in $B^{\prime}$.

Now let $A, B$ be finite decreasing filtrations of $V$. We consider filtrations $A^{\prime}, B^{\prime}$ with $\operatorname{dim} A^{p} \cap B^{q}=\operatorname{dim} A^{p} \cap B^{q}$ for all $p, q$, and show that for such filtrations, if $A^{\prime}, B^{\prime}$ are close to $A, B$ in the respective flag manifolds, then $\left(A^{\prime}, B^{\prime}\right)$ is the image of $(A, B)$ by some $g \in G L(V)$ close to the identity. Arguing as before, we may and shall assume $A=A^{\prime}$.

Fix a bigrading $C$ splitting the bifiltration $(A, B): A^{p}$ (resp. $\left.B^{q}\right)$ is the sum of the $C^{i j}$ for $i \geq p$ (resp $\left.j \geq q\right)$. Fix a basis $e_{\alpha}^{p q}$ of the $C^{p q}$. As we assumed that $\operatorname{dim} A^{p} \cap B^{q}=\operatorname{dim} A^{\prime p} \cap B^{q q}, A^{p} \cap B^{q q}$ is close to $A^{p} \cap B^{q}$ and we can find $e_{\alpha}^{p q}$ in $A^{p} \cap B^{\prime q}$ close to $e_{\alpha}^{p q}$. The endomorphism $g: e_{\alpha}^{p q} \mapsto e_{\alpha}^{p q q}$ is close to the identity, hence in $G L(V)$, and maps $A^{p} \cap B^{q}$ into, hence onto, $A^{p} \cap B^{\prime q}$. It carries $(A, B)$ to $\left(A^{\prime}, B^{\prime}\right)$.

We now apply this to mixed Hodge structures.

Proposition 3.2. Let $(W, F)$ be a mixed Hodge structure on a real vector space $V$. If a filtration $F^{\prime}$ of $V_{\mathbb{C}}$ is close to $F$ and such that $\left(W, F^{\prime}\right)$ is also a mixed Hodge structure, then there is an automorphism $g$ of $V_{\mathbb{C}}$, close to the identity, respecting $W$ and carrying $F$ to $F^{\prime}$.

Proof. By (3.1.1), we may assume that for all $w, p$, one has

$$
\operatorname{dim} W_{w} \cap F^{\prime p} \leq \operatorname{dim} W_{w} \cap F^{p}
$$

and it suffices to prove equality.

We proceed by induction on $w$. If equality in (3.2.1) holds for $w-1$, from

$$
\operatorname{dim} G r_{w}^{W}\left(F^{p}\right)=\operatorname{dim} W_{w} \cap F^{p}-\operatorname{dim} W_{w-1} \cap F^{p}
$$

we obtain

$$
\operatorname{dim} G r_{w}^{W}\left(F^{\prime p}\right) \leq \operatorname{dim} G r_{w}^{W}\left(F^{p}\right)
$$

and, because of the inductive hypothesis, equality holds if and only if it holds in (3.2.1). Taking the sum of (3.2.2) for indices $(w, p)$ and $(w, w-p-1)$, we obtain

$$
\operatorname{dim} G r_{w}^{W}\left(F^{\prime p}\right)+\operatorname{dim} G r_{w}^{W}\left(F^{\prime w-p-1}\right) \leq \operatorname{dim} G r_{w}^{W}\left(F^{p}\right)+\operatorname{dim} G r_{w}^{W}\left(F^{w-p-1}\right) .
$$

Both sides equal $\operatorname{dim} G r_{w}^{W}(V)$, implying equality in (3.2.2).

3.3. Let $\mathscr{V}$ be a polarized variation of Hodge structures on $D^{* r}$, with unipotent monodromy, corresponding to a period mapping $\Phi$ on $\mathscr{H}^{r}$, with values in the filtrations of $V_{\mathbb{C}}$.

Fix $\theta^{1}, \ldots, \theta^{d}$ in $\mathbb{R}^{r}$, with $0 \leq \theta_{i}^{1} \leq \cdots \leq \theta_{i}^{d}$ and all $\theta_{i}^{d}>0$. We want to control $\Phi(z)$ when $z$ tends to infinity in the following way: the real part $x$ is bounded, the imaginary part can be written as

$$
y=\tau_{1} \theta^{1}+\cdots+\tau_{d} \theta^{d}+b
$$


with $b$ bounded, and where, for

$$
t_{i}=\tau_{i} / \tau_{i+1}(1 \leq i<d), t_{d}=\tau_{d},
$$

one has $t_{i} \rightarrow \infty$.

For this, we approximate $\Phi$ by the nilpotent orbit $\Phi_{\text {un }}$ and apply $[3,4.20]$ to the nilpotent orbit

$$
\Phi^{*}\left(u_{1}, \ldots, u_{d}\right)=\Phi_{\mathrm{un}}\left(\sum u_{j} \theta^{j}\right)
$$

Define $T_{j}=\theta^{j} N=\sum_{i} \theta_{i}^{j} N_{i}$. Adding 1 to $u_{j}$ transforms $\Phi^{*}$ by $\exp \left(T_{j}\right)$. The $T_{j}$ are not, in general, rational. In the context of [3], where real variations are considered, this does not matter.

3.4. The $S L(2, \mathbb{R})^{d}$-theory of [3] approximates period mappings, in suitable sectors, by simpler ones which we begin by describing.

On $D^{*}$, the family of elliptic curves $\mathbb{C}^{*} / q^{\mathbb{Z}}$ gives rise, by taking $H^{1}$, to a variation of Hodge structures $\mathscr{V}$ of rank 2 and type $\{(0,1),(1,0)\}$. In a suitable basis of $V_{\mathbb{Z}}$, the corresponding period mapping

$$
\Phi: \mathscr{H} \rightarrow\left\{\text { lines in } \mathbb{R}^{2} \otimes \mathbb{C}\right\}
$$

assigns to $z \in \mathscr{H}$ the line spanned by $\left(\begin{array}{l}1 \\ z\end{array}\right)$, with

$$
\boldsymbol{\Phi}(z+1)=\left(\begin{array}{ll}
1 & 0 \\
1 & 1
\end{array}\right) \boldsymbol{\Phi}(z) .
$$

On $D^{* d}$, one can then consider real variations which are direct sum of variations of the following kind:

$$
\bigotimes_{1}^{d} \operatorname{pr}_{j}^{*} \operatorname{Sym}^{n_{j}}(\mathscr{V}) \otimes H
$$

for $H$ a fixed Hodge structure. These are the simpler variations announced. Here is an alternative description of them.

The Hodge structure $\Phi(i)$ on $\mathbb{R}^{2}$ induces a Hodge structure of weight 0 on $\mathfrak{s l}(2, \mathbb{R}) \subset \operatorname{End}\left(\mathbb{R}^{2}\right)$. Let $\rho_{j}$ be the representation of $\mathfrak{s l}(2, \mathbb{R})^{d}$ on $\mathbb{R}^{2}$ via its $j$-th factor. If $A$ is a real Hodge structure, whose underlying real vector space is a representation of $\mathfrak{s l}(2, \mathbb{R})^{d}$, and if the representation map

$$
\rho: \mathfrak{s l}(2, \mathbb{R})^{d} \rightarrow \operatorname{End}(A)
$$

is a morphism of Hodge structures, where each $\mathfrak{s l}(2, \mathbb{R})$ factor is given the Hodge structure induced by $\Phi(i)$, then $(A, \rho)$ is isomorphic to a sum of tensor products

$$
\bigotimes \operatorname{Sym}^{n_{j}}\left(\rho_{j}\right) \otimes H
$$

where $\mathbb{R}^{2}$-the representation space of $\rho_{j}$ - is given the Hodge structure $\Phi(i)$ and where $H$ is a Hodge structure with trivial action. Indeed, the isomorphism of representations of $\mathfrak{s l}(2, \mathbb{R})^{d}$ :

$$
\bigoplus \bigotimes \operatorname{Sym}^{n_{j}}\left(\rho_{j}\right) \otimes \operatorname{Hom}_{\mathfrak{s l}}\left(\bigotimes \operatorname{Sym}^{n_{j}}\left(\rho_{j}\right), A\right) \rightarrow A
$$

is compatible with Hodge structures. 
In view of our later applications, we write $\hat{T}_{j}$ for the image under the representation $\rho$ of the element

$$
\left(\begin{array}{ll}
0 & 0 \\
1 & 0
\end{array}\right)
$$

in the $j$-th factor of $\mathfrak{s l}(2, \mathbb{R})^{d}$. For $F$ the Hodge filtration of $A$, the variations we are considering correspond to period maps of the form

$$
\Phi(z)=\exp \left(\sum z_{j} \hat{T}_{j}\right) F .
$$

Denoting by $Y_{j}$ the image of the element $\left(\begin{array}{cc}1 & 0 \\ 0 & -1\end{array}\right)$ in the $j$-th factor of $\mathfrak{s l}(2, \mathbb{R})^{d}$, one has for $z$ purely imaginary

$$
\Phi(i y)=\exp \left(-\sum \log \left(y_{j}\right) Y_{j} / 2\right) F_{\sharp}
$$

where

$$
F_{\sharp}:=\Phi(i):=\Phi(i, \ldots, i) .
$$

3.5. Given a nilpotent orbit $\Phi$ on $\mathscr{H}^{d}$ and an ordering of the variables, the $S L(2, \mathbb{R})^{d}$-orbit theorem provides a period mapping $\Phi_{S L}$ of the type 3.4 which approximates it. Let $T_{j}$ be the monodromies for $\Phi$ :

$$
\Phi\left(z+e_{j}\right)=T_{j} \Phi(z),
$$

define $C(j)$ to be the cone

$$
\left\{\sum_{i=1}^{j} \lambda_{i} T_{i}, \lambda_{i}>0\right\}
$$

and $W^{j}:=W(C(j))$ as in (2.4). Define $t_{j}=y_{j} / y_{j+1}$ for $j<d$, and $t_{d}=y_{d}$. One has [3]:

(3.5.1) For $x$ bounded and $t \rightarrow \infty$, the invariant distance between $\Phi(z)$ and $\Phi_{S L}(z)$ tends to zero.

(3.5.2) The $Y_{j}$ define a $\mathbb{Z}^{d}$ grading of $V$, with $Y_{j}$ acting as multiplication by $\ell_{j}$ on $V^{\ell}, \ell=\left(\ell_{1}, \ldots, \ell_{d}\right)$. One has

$$
W_{w}^{j}=\bigoplus_{\ell_{1}+\cdots+\ell_{j} \leq w} V^{\ell}
$$

(3.5.3) The construction is compatible with tensor products. In particular, $\mathfrak{s l}(2, \mathbb{R})^{d}$ respects the polarization form.

One should beware that $\Phi$ and $\Phi_{S L}$ do not have the same transformation law for $z_{i} \mapsto z_{i}+1: \Phi$ is transformed by $\exp \left(T_{i}\right)$, while $\Phi_{S L}$ is transformed by $\exp \left(\hat{T}_{i}\right)$. The two are related as follows: $\hat{T}_{i}$ is the degree zero component, for the $Y_{j}, j<i$, of $T_{i}$. In particular,

$$
T_{1}=\hat{T}_{1} .
$$

In addition, (3.5.2) says that the monodromy weight filtration $W^{j}$ is also the monodromy weight filtration for $\sum_{1}^{j} \lambda_{i} \hat{T}_{i}$ when all $\lambda_{i}>0$. 
3.6. We now apply this to a period mapping $\Phi$ on $\mathscr{H}^{r}$, to approximate $\Phi(z)$ where $z$ is as in 3.3. With the notations of 3.3, the distance between the following pairs of Hodge structures tends to zero, when $t \rightarrow \infty$ :

(a) $\Phi(z)$ and $\Phi_{\mathrm{un}}(z)$ : by (2.3.3),

(b) $\Phi_{\mathrm{un}}(z)$ and $\Phi^{*}(i \tau)$ : one has $\Phi^{*}(i \tau)=\Phi_{\mathrm{un}}\left(i \sum \tau_{j} \theta^{j}\right)$; the hyperbolic distance between $z$ and $i \sum \tau_{j} \theta^{j}$ is in $\mathrm{O}\left(1 / \inf \left(y_{i}\right)\right)$ and one uses the distance decreasing property of period maps,

(c) $\Phi^{*}(i \tau)$ and its $S L(2, \mathbb{R})^{d}$-orbit approximation $\Phi_{S L}$ : by (3.5.1).

One concludes that the invariant distance betwen $\Phi(z)$ and $\Phi_{S L}(i \tau)$ tends to zero.

With the notations of 3.5, we set, as in (3.4.3), $F_{\sharp}=\Phi_{S L}(i)$ and define

$$
e(\tau)=\exp \left(\sum \log \left(\tau_{j}\right) Y_{j} / 2\right)
$$

It acts by multiplication by $\Pi \tau_{j}^{\ell_{j} / 2}$ on $V^{\ell}$ (notation of (3.5.2)). It respects the polarization form, hence induces an isometry of the period mapping domain. Because of (3.4.2),

$$
e(\tau) \Phi_{S L}^{*}(i \tau)=\Phi_{S L}^{*}(i)=F_{\sharp}
$$

and, consequently,

$$
e(\tau) \Phi(z) \rightarrow F_{\sharp}
$$

for $z, \tau$ as in 3.3, $t_{i} \rightarrow \infty$.

3.7. Let $I(j) \subset[1, r]$ be the set of $i$ for which $\theta_{i}^{j} \neq 0$. It is increasing with $j$. Let $C(j)$ be the cone

$$
C(j):=\left\{\sum_{i \in I(j)} \lambda_{i} N_{i}, \lambda_{i}>0\right\}
$$

and $W^{j}$ be the corresponding filtration $W(C(j))$. Those filtrations $W^{j}$ coincide with those of 3.5, for $\Phi^{*}(u)$ and the monodromies $T_{j}=\theta^{j} N$.

Some of the above results can be expressed just in terms of the $W^{j}$.

(3.7.1) There is a $\mathbb{Z}^{d}$-grading $A$, splitting all the $W^{j}: W_{w}^{j}$ is the sum of $A^{\ell}$ with $\ell_{1}+\cdots+\ell_{j} \leq w . A$ may be chosen rational and compatible with the polarization form.

Indeed, (3.5.2) gives one such grading. The statement (3.7.1) is equivalent to the statement that the $W_{w}^{j}$ generate a distributive lattice of subspaces. As the filtrations $W^{j}$ are rational, there exists a rational $\mathbb{Z}^{d}$-grading $A$ as in (3.7.1). The grading (3.5.2) is in addition compatible with the polarization form: $V^{\ell}$ orthogonal to $V^{m}$ for $\ell+m \neq 0$.

If $A, B$ are two $\mathbb{Z}^{d}$-gradings as in (3.7.1), $A^{\ell}$ and $B^{\ell}$ are both canonically isomorphic to an iterated grading of $V$ by the $G r_{w_{j}}^{W^{j}}, w_{j}=\ell_{1}+\cdots+\ell_{j}$. Order 
is irrelevant, because the $W_{w}^{j}$ generate a distributive lattice. If $g$ is the direct sum of the resulting isomorphisms $A^{\ell} \stackrel{\cong}{\longrightarrow} B^{\ell}$, one has

$$
(g-1)\left(A^{\ell}\right) \subset \bigoplus A^{m} \text { with } m \neq \ell, m_{1}+\cdots+m_{j} \leq \ell_{1}+\cdots+\ell_{j} .
$$

If we transport the grading $A$ by the polarization form, viewed as an isomorphism from $V$ to $V^{*}$, and dualize, we get another grading $A^{\prime}$, equal to $A$ if and only if $A$ is compatible with the polarization. We have $A^{\prime}=g A$ where $g$ obeys (3.7.2). The grading $g^{1 / 2} A$ is then compatible with the polarization. This shows the existence of rational gradings of the $W^{j}$, as in (3.7.1), compatible with the polarization form.

Fix any grading $A$ as in (3.7.1) and define $e_{A}(\tau)$ to be the multiplication by $\prod \tau_{j}^{\ell_{j} / 2}$ on $A^{\ell}$. If $A^{\ell}=g V^{\ell}$, with $g$ as above, $e_{A}(\tau)=g e(\tau) g^{-1}$.

Proposition 3.8. Notations being as above,

(i) $e_{A}(\tau) \Phi(z)$ tends to $g F_{\sharp}$;

(ii) $e_{A}(\tau)$ [Hodge metric at $\Phi(z)$ ] tends to the transform by $g$ of the Hodge metric at $F_{\sharp}$.

Proof. For $A$ the decomposition by the $V^{\ell}, g$ is the identity, (i) is (3.6.2), and (ii) follows as $e(\tau)$ respects the polarization form. In general,

$$
e_{A}(\tau)=g e(\tau) g^{-1}=g\left(e(\tau) g^{-1} e(\tau)^{-1}\right) e(\tau)
$$

and, because of (3.7.2), $e(\tau) g^{-1} e(\tau)^{-1}$ tends to the identity. The proposition follows.

(3.9) Remarks. (i) Fix a metric $|a|$ on each $A^{\ell}$. By 3.8 (ii), the Hodge metric at $\Phi(z)$ is comparable to the orthogonal direct sum of those metrics, multiplied by $\tau^{\ell / 2}$ :

$$
\|a\|_{\Phi(z)}^{2} \sim \sum \tau^{\ell}\left|a^{\ell}\right|^{2}
$$

i.e., the ratios of both members of (3.9.1) are bounded.

(ii) Suppose $v \in V$ is in $W_{w}^{1}$. Let $v^{1}$ be its image in $G r_{w}^{W^{1}}$. The Hodge filtration $\Phi_{\mathrm{un}}(z)$ induces a Hodge filtration on $G r_{w}^{W^{1}}$. We want to compare the Hodge norm of $v$ and $v^{1}$ at $z$. Decompose $V$ as in (3.7.1); the $A^{\ell}$ with $\ell_{1}=w$ then project to a similar decomposition $B$ of $G r_{w}^{W^{1}}$. Numbering: $B^{\ell}=0$ if $\ell_{1} \neq 0$,

$$
B^{0, \ell_{2}, \ldots, \ell_{d}}=A^{w, \ell_{2}, \ldots, \ell_{d}} .
$$

Applying (3.9.1) both to $V$ and $G r_{w}^{W^{1}}$, we obtain

$$
\left\|v^{1}\right\|^{2} \leq c \tau_{1}^{-w}\|v\|^{2}
$$

for an appropriate constant $c$. 
Proposition 3.10. Let $A$ be a real Hodge structure of weight 0 and a representation of $\mathfrak{s l}(2, \mathbb{R})$. One assumes that

$$
\rho: \mathfrak{s l}(2, \mathbb{R}) \rightarrow \operatorname{End}(A)
$$

is a morphism of Hodge structures, with $\mathfrak{s l}(2, \mathbb{R})$ being given the Hodge structure of 3.4. Let $W$ be the monodromy weight filtration for $\rho\left(\begin{array}{ll}0 & 0 \\ 1 & 0\end{array}\right)$. Then

$$
A_{\mathbb{R}} \cap W_{0} \cap F^{0} \subset A_{\mathbb{R}}^{\mathbf{s I}(2, \mathbb{R})} .
$$

Proof. $A$ can be decomposed as a direct sum

$$
\bigoplus_{n} \operatorname{Sym}^{n}\left(\mathbb{R}^{2}\right) \otimes H_{n},
$$

where $\mathbb{R}^{2}$ is the standard representation of $\mathfrak{s l}(2, \mathbb{R})$, with the Hodge structure of 3.4, and where $H_{n}$ is a Hodge structure of weight $-n$. For $e, f$ the standard basis of $\mathbb{R}^{2}, \operatorname{Sym}^{n}\left(\mathbb{R}^{2}\right)$ is the space of homogeneous polynomials of degree $n$ in $e, f, P(e, f)$ is in $W_{0}$ if it is divisible by $f^{m}, m=[(n+1) / 2]$, and in $F^{p}$ if divisible by $(e+i f)^{p}$.

We may and shall assume $A$ reduced to one of the summands (3.7.1). Take $x$ real in $W_{0} \cap F^{0}$. Choose a basis $h_{\alpha}$ of $H_{\mathbb{C}}$, compatible with the Hodge decomposition. Write $x=\sum x_{\alpha} \otimes h_{\alpha}$. If $h_{\alpha}$ is of type $(-p,-q), x_{\alpha}$ must be of type $(p, q)$, i.e., a multiple of $(e+i f)^{p}(e-i f)^{q}$. It can be in $W_{0}$ only for $n=0$.

Corollary 3.11. Let $A$ be a real Hodge structure of weight 0 and a representation of $\mathfrak{s l}(2, \mathbb{R})^{d}$. One assumes that

$$
\rho: \mathfrak{s l}(2, \mathbb{R})^{d} \rightarrow \operatorname{End}(A)
$$

is a morphism of Hodge structures, with $\mathfrak{s l}(2, \mathbb{R})$ being given the Hodge structure of 3.4. Let $W^{j}$ be the monodromy weight filtration for $\rho\left(\Delta_{j}\left(\begin{array}{ll}0 & 0 \\ 1 & 0\end{array}\right)\right)$, where $\Delta_{j}$ is the diagonal embedding of $\mathfrak{s l}(2, \mathbb{R})$ in the first $j$ factors of $\mathfrak{s l}(2, \mathbb{R})^{d}$. Then, the intersection

$$
A_{\mathrm{R}} \cap \bigcap_{j} W_{0}^{j} \cap F^{0}
$$

is contained in the $\mathfrak{s l}(2, \mathbb{R})^{d}$-invariants.

Proof. We proceed by induction on $d$. By 3.10, the intersection is contained in the invariants of the first factor $\mathfrak{s l}(2, \mathbb{R})$. This space $A^{\prime}$ is a sub-Hodge structure of $A$ acted on by $\mathfrak{s l}(2, \mathbb{R})^{d-1}$, and 3.11 follows from the induction assumption applied to $A^{\prime}$.

\section{Proof of Theorem 2.16}

4.1. In this section we prove 2.16 , and hence 2.5 , reasoning by contradiction as explained in 2.19. We fix a sequence $(z(n), u(n))$ with the following properties: $0 \leq x_{i}(n) \leq 1, \inf _{i} y_{i}(n) \rightarrow \infty, u(n) \in V_{\mathbb{Z}}$, the Hodge norm $\|u(n)\|$ of $u(n)$ at $z(n)$ is bounded, and, for some fixed $\alpha>0, u(n) \sim_{z(n)} \Phi^{0}(z(n))$ (notation of 2.15). 
We have to show that for a suitable subsequence,

(4.1.1) $u(n)$ is constant, and

(4.1.2) its constant value is in $W_{0}$ as well as in $F^{0}$ for some limiting Hodge filtration $F$.

Taking a subsequence, we may and shall assume that for suitable $\theta^{1}, \ldots, \theta^{d}$ in $\mathbb{R}^{r}$, one has

$$
y(n)=\tau_{1}(n) \theta^{1}+\cdots+\tau_{d}(n) \theta^{d}+b(n)
$$

with $t_{j}(n):=\tau_{j}(n) / \tau_{j+1}(n) \rightarrow \infty, t_{d}(n)=\tau_{d}(n) \rightarrow \infty$, and $b(n)$ bounded. Then, in addition to (4.1.1) and (4.1.2), we will show that, with $v:=u(n)$ and $T_{j}:=\sum \theta_{j}^{i} N^{i}$,

$$
T_{j} v=0
$$

The proof is by induction on $d \geq 1$.

4.2. If $\theta^{j}$ is a linear combination of the preceding $\theta^{i}$ (this includes the case $\left.j=1, \theta^{1}=0\right)$, (4.1.3) can be replaced by a similar expansion with $\theta^{j}$ omitted. This lowers $d$ and the claims (4.1.1), (4.1.2), (4.1.4) are unaffected: by induction, we may and shall assume that the $\theta^{j}$ are linearly independent.

We assumed that $\inf _{i} y_{i}(n)$ tends to $\infty$. This means that for each $i$, one of $\theta_{i}^{1}, \ldots, \theta_{i}^{d}$, is nonzero and that the first to be nonzero is positive.

The $\theta$ 's are not uniquely determined by $y(n)$-only the flag $\left\langle\theta^{1}\right\rangle$ $\subset\left\langle\theta^{1}, \theta^{2}\right\rangle \subset \cdots$ is. Adding to $\theta^{2}, \ldots, \theta^{d}$ a large enough multiple of $\theta^{1}$, then to $\theta^{3}, \ldots, \theta^{d}$ a large enough multiple of $\theta^{2}$, etc., we may and shall assume that $\theta^{1} \leq \theta^{2} \leq \cdots \leq \theta^{d}$.

For simplicity of notation, we will reorder the coordinates $z_{i}$ so that the $i$ for which the $i$-th coordinate of $\theta^{j}$ is not zero form an initial segment $1 \leq i \leq a(j)$. We let $W^{j}$ be the monodromy weight filtration $W(C(j))$, for the cone

$$
C(j)=\left\{\sum_{1}^{a(j)} \lambda_{i} N_{i}: \lambda_{i}>0\right\} .
$$

Let $\Phi^{\prime}:=\Phi_{\mathrm{un},[1, a(1)]}$ be the nilpotent orbit in the $z_{i}, i \leq a(1)$, approximating $\Phi$. By 2.3, and the fact that $\inf _{i \leq a(1)}\left(y_{i}(n)\right)$ is comparable to $\sup \left(y_{i}(n)\right)$, we still have $u(n)$ bounded in the $\Phi^{\prime}$-Hodge norm at $z(n)$, and $\sim_{z(n)} \Phi^{\prime 0}(z(n))$. The monodromies, as well as the limiting Hodge filtrations, being the same for $\Phi$ and $\Phi^{\prime}$, we may replace $\Phi$ by $\Phi^{\prime}$ : we may and shall assume that $\Phi$ is a nilpotent orbit in $z_{1}, \ldots, z_{a(1)}$. It follows that $\left(W^{1}, \Phi\right)$ is a mixed Hodge structure.

For each $w, G r_{w}^{W^{1}}$ is a variation of Hodge structures of weight $w$. It is independent of $z_{1}, \ldots, z_{a(1)}$ : if $a(1)=r$, it is a constant Hodge structure. 
If $a(1)<r$, it corresponds to a period map $\Phi_{1}$ defined on $\mathscr{H}^{[a(1)+1, r]}$. Let $z(n)^{1}$ be the projection of $z(n)$ on $\mathscr{H}^{[a(1)+1, r]}$. The projection of $\theta^{1}$ being zero, (4.1.3) projects to

$$
y(n)^{1}=\tau_{2}(n) \bar{\theta}^{2}+\cdots+\tau_{d}(n) \bar{\theta}^{d}+\bar{b}(n)
$$

with ${ }^{-}$denoting projection. This is an expansion like (4.1.3), but with $d-1$ $\theta$ 's. This is one of two mechanisms by which induction will proceed.

We polarize $G r_{w}^{W^{1}}(V)$ using any rational element $N$ in $C(1)$ : it polarizes the mixed Hodge structure, inducing a polarization of the grading.

Our first task is to prove:

Proposition 4.3. For $n$ large enough, $u(n)$ is in $W_{0}^{1}$.

We say that $u \in V$ is in the position $\ell=\left(\ell_{1}, \ldots, \ell_{d}\right)$ relative to the filtrations $W^{j}$ if, for $A$ a $\mathbb{Z}^{d}$-grading splitting the $W^{j}, \ell$ is the largest multi-index, in the lexicographic order, for which the $\ell$-component of $u$ does not vanish. By (3.7.2), this does nor depend on the choice of $A$.

If $u$ is in position $\left(\ell_{1}, \ldots, \ell_{d}\right)$, then it is in $W_{\ell_{1}}^{1}$, with a nonzero image $u^{1}$ in $G r_{\ell_{1}}^{W^{1}}(V)$, and $u^{1}$ is in the position $\left(\ell_{2}, \ldots, \ell_{d}\right)$ relative to the filtrations induced by $W^{2}\left[\ell_{1}\right], \ldots, W^{d}\left[\ell_{1}\right]$, where $W[m]_{w}=W_{m+w}$.

Those induced filtrations are also the monodromy weight filtrations for the action of any $N \in C(j)$ on $G r_{\ell_{1}}^{W^{1}}(V)$ : this expresses the fact that $W^{j}$ is the relative monodromy weight filtration of $W^{1}$ and any $N \in C(j)([1,(3.3)])$.

Taking a subsequence, we may and shall assume that all $u(n)$ are in the position $\left(\ell_{1}, \ldots, \ell_{d}\right)$, for a suitable $\left(\ell_{1}, \ldots, \ell_{d}\right)$.

Lemma 4.4. For each $j$, one has $\ell_{1}+\cdots+\ell_{j} \geq 0$.

Proof. We will prove by induction on $d$ the following more general statement: one takes $\Phi$ to be a variation of weight $w \geq 0 ; z, u, \theta, \tau$ are as before (except that the $\theta$ are not assumed linearly independent); the $u(n)$ are in position $\left(\ell_{1}, \ldots, \ell_{d}\right)$ relative to the $W^{j}$; one assumes $u(n) \sim_{z} \Phi^{0}$ and

$$
\|u(n)\| \leq c \tau_{1}(n)^{-w / 2}
$$

One claims

$$
w+\ell_{1}+\cdots+\ell_{j} \geq 0 \quad \text { for each } j .
$$

If $\theta^{j}$ is a linear combination of previous $\theta$, we have $W^{j}=W^{j-1}$ (resp. $W^{j}$ trivial if $j=1, \theta^{1}=0$ ) and $\ell_{j}=0$. Reasoning as in 4.2, we may, using the induction hypothesis, assume that the $\theta$ are linearly independent.

As in 4.2, we may and shall assume that $\Phi$ is a nilpotent orbit in the variables $z_{1}, \ldots, z_{a(1)}$. Let $\Phi_{1}$ be the period mapping on $\mathscr{H}^{[a(1)+1, r]}$ corresponding to $G r_{\ell_{1}}^{W^{1}}(V)$ (weight $w+\ell_{1}$ ) and let $u(n)^{1}$ be the image of $u(n)$ in this grading. 
Lemma 4.5. Assumptions and notations being as above, we have

(i) $w+\ell_{1} \geq 0$,

(ii) $\quad\left\|u(n)^{1}\right\|^{2} \leq c \tau_{1}(n)^{-w-\ell_{1}} \quad$ (Hodge norm ),

(iii) $u(n)^{1} \sim_{z} \Phi_{1}^{0}\left(z(n)^{1}\right)$.

Proof. Let us transform $u(n) \sim_{z} \Phi^{0}(z(n))$ by $e(\tau(n)$ ) (notations of 3.6). In the Hodge norm for $e(\tau(n)) \Phi(z(n))$, we continue to have

$$
e(\tau(n)) u(n) \sim_{z} e(\tau(n)) \Phi^{0}(z(n))
$$

The subspace on the right tends to $F_{\sharp}^{0}$. Any limiting value of the ray spanned by the real vector $e(\tau(n)) u(n)$ is hence in

$$
W_{w+\ell_{1}}^{1} \cap F_{\sharp}^{0} \cap \bar{F}_{\sharp}^{0} .
$$

As $\left(W^{1}[w], F_{\sharp}\right)$ is a mixed Hodge structure, this intersection can be nonzero only for $w+\ell_{1} \geq 0$. This proves (i).

Comparing the asymptotics of the Hodge norm for $V$ and for $G r_{\ell}^{W^{1}}$, one finds (cf. 3.9) that for any $v \in W_{\ell}^{1}$, with image $v^{1}$ in the grading, one has at $z(n)$

$$
\left\|v^{1}\right\|^{2} \leq c \tau_{1}(n)^{-\ell_{1}}\|v\|^{2}
$$

proving (ii).

For (iii), we will consider angles in a fixed metric. As explained in 2.17(ii), we still have $u(n) \sim_{z} \Phi^{0}$, and it suffices to prove $u(n)^{1} \sim_{z} \Phi_{1}^{0}$, in this new sense.

Fix $z_{0}$ with big enough imaginary part so that $\left(W^{1}, \Phi_{\text {un }}\left(z_{0}\right)\right)$ is a mixed Hodge structure. As $\exp (-z(n) N)$ is bounded by some $\sup \left(y(n)_{i}\right)^{k}$, we have

$$
\exp \left(-\left(z(n)-z_{0}\right) N\right) u(n) \sim_{z} \exp \left(-\left(z(n)-z_{0}\right) N\right) \Phi^{0}(z(n)) .
$$

The filtration $\exp \left(-\left(z(n)-z_{0}\right) N\right) \Phi(z(n))$ tends to $\Phi_{\text {un }}\left(z_{0}\right)$. Together with $W^{1}$, both filtrations define a mixed Hodge structure. It follows that some complex endomorphism $\gamma_{n}$ tending to 1 respects $W^{1}$, transforms the latter into the former, and satisfies

$$
v(n):=\gamma_{n}^{-1} \exp \left(-\left(z(n)-z_{0}\right) N\right) u(n) \sim_{z} \Phi_{\mathrm{un}}^{0}\left(z_{0}\right)
$$

As $v(n) \in W_{\ell_{1}}^{1}$, one also has

$$
v(n) \sim_{z} W_{\ell_{1}}^{1} \cap \Phi_{\mathrm{un}}^{0}\left(z_{0}\right)
$$

The projection $v(n)^{1}$ of $v(n)$ in $G r_{\ell_{1}}^{W^{1}}$ is the transform of a nonzero element of $G r_{\ell_{1}}^{W^{1}}\left(V_{\mathbb{Z}}\right)$ by $\gamma_{n}^{-1} \exp \left(-\left(z(n)-z_{0}\right) N\right)$. Hence, its size is at least like $c \sup \left(y(n)_{i}\right)^{-k}$, while that of $v(n)$ is at most like $c \sup \left(y(n)_{i}\right)^{k}$. It follows that 
the exponential closeness $(4.5 .1)$ continues to hold $\bmod W_{\ell_{1}-1}^{1}$ :

$$
v(n)^{1} \sim_{z} \Phi_{\mathrm{un}}^{0}\left(z_{0}\right) \text { in } G r_{\ell_{1}}^{W^{1}}(V) .
$$

Applying $\exp \left(\left(z(n)-z_{0}\right) N\right) \gamma_{n}$, we get 4.5(iii).

Proof of 4.4. If $a(1)=r$, one has $W^{j}=W^{1}$ for all $j, \ell_{j}=0$ for $j \geq 2$, and 4.4 follows from $4.5(\mathrm{i})$. If $a(1)<r$, we apply the induction hypothesis to $\Phi_{1}$, of weight $w+\ell_{1} \geq 0$ (cf. $4.5(\mathrm{i})$ ), to $u(n)^{1}$ and to $z(n)^{1}$, which has an expansion like (4.1.3), with $d-1 \theta$ 's. By 4.5(ii), (iii), the required estimates hold.

Proof of 4.3. Fix a rational decomposition $V=\bigoplus_{\mathbb{Z}^{d}} A^{\ell}$ as in (3.7.1). The projection of $V_{\mathbb{Z}}$ in $A^{\ell}$ is a lattice. By (3.9.1), it follows that if $u(n)$ has a nonzero projection in $A^{a}$, then

$$
\|v(n)\|^{2}>>\tau_{1}(n)^{a_{1}} \cdots \tau_{d}(n)^{a_{d}} .
$$

Take $a=\left(\ell_{1}, \ldots, \ell_{d}\right)$. By definition, the projection of $v(n)$ is nonzero. One has

$$
\tau_{1}(n)^{\ell_{1}} \cdots \tau_{d}(n)^{\ell_{d}}=\left(\frac{\tau_{1}(n)}{\tau_{2}(n)}\right)^{\ell_{1}}\left(\frac{\tau_{2}(n)}{\tau_{3}(n)}\right)^{\ell_{1}+\ell_{2}} \cdots \tau_{d}(n)^{\ell_{1}+\cdots+\ell_{d}} .
$$

As $\|v(n)\|$ is bounded, we conclude that

$$
\ell_{1}=\ell_{2}=\cdots=\ell_{d}=0 \text {. }
$$

In particular, $\ell_{1}=0$, proving 4.3 .

4.6. We now apply the induction hypothesis to $G r_{0}^{W^{1}}(V)$, corresponding to a period mapping $\Phi_{1}$, and to the projection $u(n)^{1}$ of $u(n)$ in $G r_{0}^{W^{1}}(V)$.

If $a(1)=r$, the Hodge structure $G r_{0}^{W^{1}}(V)$ is constant. By 4.5(ii), or directly by 3.9(ii), $u(n)^{1}$ is bounded. Being in a lattice, it can take only a finite number of values. We may and shall assume it is constant and define $u^{1}:=u(n)^{1}$. As $u^{1} \sim_{z} \Phi_{1}^{0}$, one has $u^{1} \in \Phi_{1}^{0}$.

If $a(1)<r$, the induction hypothesis applies by (4.2.1) and 4.5. Again, we find that $u(n)^{1}$ may and shall be assumed to have a constant value $u^{1}$. By (4.1.4), $u^{1}$ is killed by $T_{j}(j \geq 2)$ and in particular sits in the $W_{0}^{j} \quad(j \geq 2)$. It is also in $F^{0}$ for some limiting Hodge filtration $F$ (all this in $G r_{0}^{W^{1}}(V)$ ).

$T_{1}$ maps $W_{0}^{1}$ to $W_{-2}^{1}$, inducing a morphism of Hodge structures

$$
G r_{0}^{W^{1}}(V) \rightarrow G r_{-2}^{W^{1}}(V)(-1)
$$

We next prove

Proposition 4.7. $u^{1}$ is in the kernel of the morphism (4.6.1).

Proof. If we transform the assumption

$$
u(n) \sim_{z} \Phi^{0}
$$


by $e(\tau(n))$ (notations of (3.6.1)), we obtain

$$
e(\tau(n)) u(n) \sim_{z} e(\tau(n)) \Phi^{0}(z(n))
$$

where angles are now taken in the Hodge metric for $e(\tau(n)) \Phi(z(n))$. The Hodge norm of $e(\tau(n)) u(n)$ in this metric - equal to that of $u(n)$ in the Hodge metric for $\Phi(z(n))$ - is bounded by assumption. The $e(\tau(n)) \Phi(z(n))$ tend to the Hodge filtration $F_{\sharp}$ (cf. 3.6). The Hodge metric for $e(\tau(n)) \Phi(z(n))$ tends to that for $F_{\sharp}$. It follows that $e(\tau(n)) u(n)$ remains bounded and, taking a subsequence, we may and shall assume that it has a limit $u_{0}$. We have

$$
u_{0} \in F_{\sharp}^{0} .
$$

Consider the decomposition $V=\bigoplus_{\mathbb{Z}^{d}} V^{a}: e(\tau)$ acts on $V^{a}$ as multiplication by $\tau^{a / 2}$.

By 3.10, $u_{0}$ is in the sum of the $V^{\ell}$ with $\ell_{1}=0$ and is in the kernel of $\hat{T}_{1}$. The components $u(n)^{(\ell)}$ of $u(n)$, for $\ell_{1}=0$, depend only on $u^{1}$ : they are independent of $n$. That $u^{1}$ is killed by the $T_{j}(j \geq 2)$ implies it is in the $W_{0}^{j}$ and that, for $\ell_{1}=0, u(n)^{(\ell)}$ can be nonzero only for $\ell_{2}+\cdots+\ell_{j} \leq 0$. The 0-component $u(n)^{(0)}$ coincides with $u_{0}$, killed by $\hat{T}_{1}=T_{1}$ (3.5.4).

The $V^{\ell}$ with $\ell_{1}=-2$ project to a decomposition of $G r_{-2}^{W^{1}}(V)$. Applying $T_{1}$ to the $u(n)^{(\ell)}$ for $\ell_{1}=0$, we find that $T_{1} u^{1}$ has a nonzero component in $V^{\ell}\left(\ell_{1}=-2\right)$ only when $\left(\ell_{2}, \ldots, \ell_{d}\right) \neq(0, \ldots, 0), \ell_{2}+\cdots+\ell_{j} \leq 0$.

The $e(\tau(n)) \Phi(z(n))$ belong to a compact family of Hodge filtrations $F$, for which $\left(W^{1}, F\right)$ is mixed Hodge. By 3.2, $e(\tau(n)) u(n)$ is a sum $\chi+\epsilon, \chi$ in $e(\tau(n)) \Phi^{0},\|\epsilon\| \leq \exp \left(-\alpha \sup _{i} y_{i}(n)\right)$. By compacity, or 3.2, $\chi$ can be taken in $W^{1} \cap F$. Apply $T_{1}$; by (3.5.4),

$$
e(\tau(n)) T_{1} u(n)=\tau_{1}(n)^{-1} T_{1}(e(\tau(n)) u(n))=\tau_{1}(n)^{-1}\left(T_{1} \chi+T_{1} \epsilon\right)
$$

and we find that $T_{1} u^{1}$ is the sum of an element in $\Phi^{-1}(z(n))$ plus an exponentially small term. Passing to the limit, we obtain

$$
T_{1} u^{1} \in F_{\sharp}^{-1},
$$

where $F_{\sharp}$ is the filtration in $G r_{-2}^{W^{1}}(V)$ induced by $F_{\sharp}$. If we go to $G r_{-2}^{W^{1}}(V)(-1)$, to have a Hodge structure of weight zero, this reads

$$
T_{1} u^{1} \in F_{\sharp}(-1)^{0} \text {. }
$$

The action of the individual factors of $S L(2, \mathbb{R})^{d}$ of index $\neq 1$, and the filtration $F_{\sharp}(-1)$ on $G r_{-2}^{W^{1}}(V)(-1)$, are of the type considered in 3.11. By 3.11, $T_{1} u^{1}$ is in $V^{-2,0, \ldots, 0}$, hence it is zero as the $(-2,0, \ldots, 0)$ component has been shown to vanish. This proves 4.7 . 
Proposition 4.8. $T_{1} u(n)$ is exponentially small: of Hodge norm

$$
<\exp \left(-\beta \sup \left(y_{i}(n)\right)\right)
$$

for suitable $\beta>0$.

Proof. By 4.7, $T_{1} u(n)$ is in $W_{-3}^{1}$. It is also the sum of $\chi$ in $\Phi^{-1}(z(n))$ and of an exponentially small $\epsilon$. The same holds after applying $e(\tau(n))$. We gain that the $e(\tau(n)) \Phi(z(n))$ belong to a compact family of filtrations $F$, for which

$$
W_{-3}^{1} \cap F^{-1} \cap \bar{F}^{-1}=0,
$$

so that $e(\tau(n)) T_{1} u(n)$ must be exponentially small. Hence so is $T_{1} u(n)$.

4.9. We now complete the proof of 2.16. With angles measured using a fixed metric, we have

$$
u(n) \sim_{z} \Phi^{0}(z(n)) .
$$

Applying $\exp \left(-i \tau_{1}(n) T_{1}\right)$, of polynomial size in $\sup _{i}\left(y_{i}(n)\right)$ :

$$
\exp \left(-i \tau_{1}(n) T_{1}\right) u(n) \sim_{z} \Phi^{0}\left(z(n)-i \tau_{1}(n) \theta^{1}\right)
$$

and, by 4.8 ,

$$
u(n) \sim_{z} \Phi^{0}\left(z(n)-i \tau_{1}(n) \theta^{1}\right) .
$$

If $d=1$, we choose the expansion (4.1.3) (subtracting a constant to $\tau_{1}(n)$ ) so that $y_{j}(n)-\tau_{1}(n) \theta_{j}^{1} \geq A>0$. As $u(n)$ is close to $\Phi^{0}$ at $z(n)$ and at $z(n)-i \tau_{1}(n) \theta^{1}$, its Hodge norm at both places is close to $Q(u(n), u(n))^{1 / 2}$ and hence is bounded. Moreover, $z(n)-i \tau_{1}(n) \theta^{1}$ remains bounded and the corresponding Hodge filtrations remain in a compact set. Being bounded and integral, $u(n)$ can take only finitely many values. Taking a subsequence for which $z(n)-i \tau_{1}(n) \theta^{1}$ tends to a limit, we find that $u$ is in the corresponding $\Phi^{0}$. It is in $W_{0}$ by 4.3. This proves (4.1.2), while (4.1.4) results from 4.7.

Assume now $d>1$. The imaginary part of $z(n)-i \tau_{1}(n) \theta^{1}$ is

$$
\tau_{2}(n) \theta^{2}+\cdots+\tau_{d}(n) \theta^{d}+b(n),
$$

an expansion as in (4.1.3), but with only $d-1 \theta$ 's. Applying the induction assumption, we see that, on a subsequence, $u(n)$ satisfies (4.1.1) and (4.1.2) and is killed by the $T_{j}(j>1)$. Being constant, it is also killed by $T_{1}$ (cf. 4.8). This finishes the proof.

\section{REFERENCES}

[1] E. Cattani and A. Kaplan, Polarized mixed Hodge structures and the local monodromy of a variation of Hodge structure, Invent. Math. 67 (1982), 101-115.

[2] Degenerating variations of Hodge structure, Actes du Colloque de Théorie de Hodge, Luminy 1987. Astérisque 179-180 (1989), 67-96.

[3] E. Cattani, A. Kaplan, and W. Schmid, Degeneration of Hodge structures, Ann. of Math. (2) 123 (1986), 457-535.

[4] P. Deligne, Equations différentielles à points singuliers réguliers, Lecture Notes in Math., vol 163, Springer-Verlag, Berlin and New York, 1970. 
[5] P. Griffiths, ed., Topics in transcendental algebraic geometry, Ann. of Math. Stud., no. 106, Princeton Univ. Press, Princeton, NJ, 1984.

[6] W. Schmid, Variations of Hodge structure: the singularities of the period mapping, Invent. Math. 22 (1973), 211-319.

[7] A. Weil, Abelian varieties and the Hodge ring, Andre Weil: Collected Papers III, SpringerVerlag, Berlin and New York, 1979, pp. 421-429.

ABSTRACT. Let $S$ be a nonsingular complex algebraic variety and $\mathscr{V}$ a polarized variation of Hodge structure of weight $2 p$ with polarization form $Q$. Given an integer $K$, let $S^{(K)}$ be the space of pairs $(s, u)$ with $s \in S$, $u \in \mathscr{V}_{s}$ integral of type $(p, p)$, and $Q(u, u) \leq K$. We show in Theorem 1.1 that $S^{(K)}$ is an algebraic variety, finite over $S$. When $\mathscr{V}$ is the local system $H^{2 p}\left(X_{s}, \mathbb{Z}\right) /$ torsion associated with a family of nonsingular projective varieties parametrized by $S$, the result implies that the locus where a given integral class of type $(p, p)$ remains of type $(p, p)$ is algebraic.

(E. Cattani and A. Kaplan) Department of Mathematics and Statistics, University of MASSACHUSETTS, AMHERST, MassachusetTs 01003

E-mail address, E. Cattani: cattani@math.umass .edu

E-mail address, A. Kaplan: kaplan@math.umass .edu

(P. Deligne) School of Mathematics, Institute for Advanced Study, Princeton, New JERSEY 08540 